\title{
Feedback and environmental effects in elliptical galaxies
}

\author{
Craig L. Sarazin ${ }^{1}$ \\ ${ }^{1}$ Department of Astronomy, University of Virginia, P.O. Box 400325, Charlottesville, VA \\ 22904-4325, USA \\ email: sarazin@virginia.edu
}

\begin{abstract}
The role of the environment of an elliptical galaxy on its hot interstellar gas is discussed. In general, the X-ray halos of early-type galaxies tend to be smaller and fainter in denser environments, with the exception of group-central galaxies. X-ray observations show many examples of nearby galaxies which are undergoing gas stripping. On the other hand, most bright galaxies in clusters do manage to retain small coronae of X-ray emission. Recent theoretical and observational results on the role of feedback from AGN at the centers of elliptical galaxies on their interstellar gas are reviewed. X-ray observations show many examples of X-ray holes in the central regions of brightest-cluster galaxies; in many cases, the X-ray holes are filled with radio lobes. Similar radio bubbles are seen in groups and individual early-type galaxies. "Ghost bubbles" are often seen at larger radii in clusters and galaxies; these bubbles are faint in high radio frequencies, and are believed to be old radio bubbles which have risen buoyantly in the hot gas. Low frequency radio observations show that many of the ghost bubbles have radio emission; in general, these long wavelength observations show that radio sources are much larger and involve greater energies than had been previously thought. The radio bubbles can be used to estimate the total energy output of the radio jets. The total energies deposited by radio jets exceed the losses from the gas due to radiative cooling, indicating that radio sources are energetically capable of heating the cooling core gas and preventing rapid cooling.
\end{abstract}

Keywords. galaxies: clusters: general - cooling flows - galaxies: elliptical and lenticular, cD — galaxies: halos — galaxies: ISM — X-rays: galaxies — X-rays: galaxies: clusters

\section{Environmental Effects on X-ray Emission}

Given the wide range in X-ray luminosities of early-type galaxies of a given optical luminosity, the question naturally arises as to whether part of this dispersion might be due to the effects of environment on their X-ray emission. White \& Sarazin (1991) suggested that elliptical galaxies in dense environments were fainter than those in sparse regions. In this and most subsequent studies, the local density was characterized by the projected galaxy density in the region around the target galaxy. Brown \& Bregman (2000) argued that early-type galaxies in dense regions were more luminous; their sample included a number of group-center ellipticals. Some studies, mainly using ROSAT data, found no correlation e.g., O'Sullivan et al. 2001. More recent Chandra studies of groups and clusters (e.g, Jeltema et al. 2008; Sun et al. (2007)) seem to confirm a general anti-correlation of local density and X-ray luminosity. Observations of several galaxies in nearby clusters show evidence for ram pressure stripping; examples include M86 (Randall et al. 2008) and NGC 4552 (Machacek et al. 2006). However, despite the efficiency of stripping, most bright early-type galaxies in clusters do retain small coronae (Sun et al. (2007)).

\section{AGN Feedback in Early-Type Galaxies}

In recent years, evidence has been found for a coupling between supermassive black holes (SMBHs) in the centers of galaxies, and their galaxy hosts. First, the masses of the SMBHs 
are proportional to the bulge mass of the host, suggesting that star formation and SMBH accretion are connected. Second, the luminosity function of galaxies falls below that expected for dark matter halos at high masses in a way that can be understood if AGN suppress star formation in massive galaxies. Finally, less gas cools to low temperatures at the centers of cool core clusters, groups, and individual ellipticals than expected unless something heats the gas, and AGNs are the leading candidates. In cool core clusters, X-ray deficits ("radio bubbles") have been found at the locations of the lobes of the radio sources associated with the brightest cluster galaxies (BCGs; Fabian et al. 2006; Blanton et al. 2001); similar radio bubbles are seen in groups and individual elliptical galaxies. "Ghost bubbles"' at larger radii are also seen which lack high frequency radio emission. These are thought to be older radio bubble which have risen buoyantly through the hot gas. Recent low frequency radio observation often show that the ghost bubbles are filled with long wavelength radio emission. Recent examples include Abell 2597 (Clarke et al. 2005) and Abell 262 (Clarke et al. 2009).

Radio bubbles in clusters and ellipticals allow the determination of the total kinetic energy output of the radio jets. The energy injected by the jets must at least equal the work needed to inflate the bubbles plus the internal energy associated with their pressure plus any energy in shocks in the surrounding medium. In general, these energies greatly exceed the synchrotron radio emission from the AGN, and the energies in the radio lobes are much greater than the values expected from equipartition. These estimates indicate that radio jets in cluster BCGs and ellipticals deposit enough energy to balance radiative cooling in these systems (Dunn \& Fabian 2006; Rafferty et al. 2006). The mechanisms by which the radio sources heat the X-ray gas are uncertain, although heating by sound waves and weak shocks is a possibility. Recent observations of Abell 2052 (Blanton et al. 2009) show X-ray ripples which are similar to the features seen in the Perseus cluster.

\section{Acknowledgements}

I thank Liz Blanton, Tracy Clarke, Judith Croston, and Ming Sun for comments and help with the figures in the talk. This work was supported by NASA Chandra grants GO7-8078X, GO7-8081A, GO8-9085X, GO8-9083X, NASA Herschel grant RSA1373266, and NASA HST grant HST-GO-10597.03-A.

\section{References}

Blanton, E. L., Sarazin, C. L., McNamara, B. R., \& Wise, M. W. 2001, ApJ 558, L15

Blanton, E. L. et al. 2009, ApJ 697, L95

Brown, B. A. \& Bregman, J. N. 2000, ApJ 539, 592

Clarke, T. E. et al. 2009 ApJ 625, 748

Clarke, T. E. et al. 2009 ApJ 697, 481

Dunn, R. J. H. \& Fabian, A. C. 2006, MNRAS 373, 959

Fabian, A. C. et al. 2006, MNRAS 366, 417

Jeltema, T. E., Binder, B., \& Mulchaey, J. S. 2008, ApJ 679, 1162

Machacek, M., Nulsen, P. E. J., Jones, C., \& Forman, W. R. 2006 ApJ 648, 947

O'Sullivan, E., Forbes, D. A., \& Ponman, T. J. 2001, MNRAS 328, 461

Randall, S. et al. 2008, ApJ 688, 208

Rafferty, D., McNamara, B. R., Nulsen, P. E. J., \& Wise, M. W. 2006, ApJ 652, 216

Sun, M., Jones, C., Forman, W., Vikhlinin, A., Donahue, M., \& Voit, M. 2007, ApJ 657, 197

White, R. E. III \& Sarazin, C. L. 1991, ApJ 367, 476 